\title{
A Mild and Efficient Synthesis of 4-Quinolones and Quinolone Heterocycles
}

Daniel Zewge,* Cheng-yi Chen, Curtis Deer, ${ }^{\dagger}$ Peter G. Dormer, and Dave L. Hughes Department of Process Research, Merck Research Laboratories, P.O.Box 2000, New Jersey 07065

Daniel_Zewge@merck.com

\section{Supporting Information-1}

\section{Table of contents}

General Experimental Methods for compounds 1 - 9.

S2 -S3

Table-1 and Characterization Data (ESI-HRMS and ${ }^{1} \mathrm{H} /{ }^{13} \mathrm{C}$ NMR)

S3 - S6

for compounds $1-9$.

Table-2 and General Experimental Methods for compounds 10 - 12.

S6 -S7

Characterization Data (ESI-HRMS and ${ }^{1} \mathrm{H} /{ }^{13} \mathrm{C}$ NMR) for compounds 10-12.

S7 - S8

Table-3 and General Experimental Methods for compounds 13 - 16.

S8 - S9

Characterization Data (ESI-HRMS and ${ }^{1} \mathrm{H} /{ }^{13} \mathrm{C}$ NMR) for compounds 13- 16.

S9-S10

Table-4 and General Experimental Methods for compounds 17 - 26.

S10-S11

Characterization Data (ESI-HRMS and ${ }^{1} \mathrm{H} /{ }^{13} \mathrm{C}$ NMR) for compounds 17- 26.

$\mathrm{S} 12-\mathrm{S} 16$ 
Experimental Section: Unless otherwise noted reactions were carried out under $N_{2}$ atmosphere in predried glassware. All solvents and aniline starting materials were purchased from commercial sources and were used as received. ${ }^{1} \mathrm{H}$ NMR $(400 \& 500 \mathrm{MHz})$ and ${ }^{13} \mathrm{C}$ NMR $(100 \& 125 \mathrm{MHz})$ chemical shifts are referenced to tetramethylsilane. Coupling constants $(J)$ are given in Hz. ESI-HRMS results were collected using a mass spectrometer that was operated at a resolution of 10,000 using an electrospray interface and a liquid carrier of $50 \%$ aqueous $10 \mathrm{mM}$ ammonium acetate and $50 \%$ acetonitrile. A sample concentration of $50 \mu \mathrm{g} / \mathrm{mL}$ was used. $1 \mu \mathrm{L}$ of sample was injected into the carrier stream.

\section{General Procedure for preparation of enamine diester adducts (Table 1, 1a-9a) and (Table 3,} 13a - 16a). To a solution of $o$-anisidine $(1 \mathrm{~g}, 8.1 \mathrm{mmol})$ in $\mathrm{MeOH}(10 \mathrm{~mL})$ at $5{ }^{\circ} \mathrm{C}$ was added dimethylacetylenedicarboxylate (DMAD), (1.4g, $9.7 \mathrm{mmol}, 1.2 \mathrm{eq})$. The mixture was aged at $\mathrm{rt}$ over 2h. The product precipitated during the aging process. The yellow solid was filtered and washed with MTBE $(10 \mathrm{~mL})$ followed by heptane $(20 \mathrm{~mL})$. Enamine diester 1a was isolated as a yellow solid in 93\% yield (2g, $7.5 \mathrm{mmol})$. Crude solid was directly used for the subsequent cyclization reaction. This procedure was used for the preparation of bis-enamine adducts (Table 3, 13a-16a), with DMAD (2.5 eq) and for the preparation of heterocyclic adducts 17a, 17c, 18a, 20a, 22a, 23a, and 26a.

2. General Procedure for the preparation of 4-quinolones $(1 b-9 b)$, and $(13 b-16 b)$. Enamine diester 1a $(1.8 \mathrm{~g}, 6.6 \mathrm{mmol})$, was added to a RBF that was equipped with a thermocouple and a stirrer. Eaton's reagent $(7 \mathrm{~mL})$ was then added to the flask. Reaction mixture was aged at $50{ }^{\circ} \mathrm{C}$, and progress of reaction was followed by HPLC. HPLC conditions: Zorbax RX-C8 column, 150 x 3 mm, gradient method, $\mathrm{ACN}: 0.1 \% \mathrm{H}_{3} \mathrm{PO}_{4}$ 15:85 over $20 \mathrm{~min}$ hold $5 \mathrm{~min}$ at 80:20 then to 15:85 over $1 \mathrm{~min}$. Flow rate $=1 \mathrm{~mL} / \mathrm{min}, \lambda=210 \mathrm{~nm}$. Retention time, $\mathbf{1 a}=18.0 \mathrm{~min}, \mathbf{1} \mathbf{b}=12.2 \mathrm{~min}$. When conversion was $\geq 98 \%$, reaction mixture was cooled to $5{ }^{\circ} \mathrm{C}$ and slowly transferred to an excess saturated sodium carbonate solution (10 eq.) that was cooled to $10^{\circ} \mathrm{C}$. Solid was filtered and washed with water $(20 \mathrm{~mL})$ and was dried in a vac. oven at $50{ }^{\circ} \mathrm{C}$ for $12 \mathrm{~h}$. 1 b was isolated in $97 \%$ yield $(1.5 \mathrm{~g}, 6.4 \mathrm{mmol})$ and $98 \mathrm{wt} \%$. This 
procedure was used for the preparation of bis-quinolones (Table 3, 13b-16b) and heterocyclic quinolones (17b, 17d, 18b, 20b, 22b, 23b, and 26b)

Table 1: Synthesis of 2-Carboxy-4-Quinolones

Entry Reactant $\quad$ Product $\quad \%$ Yield

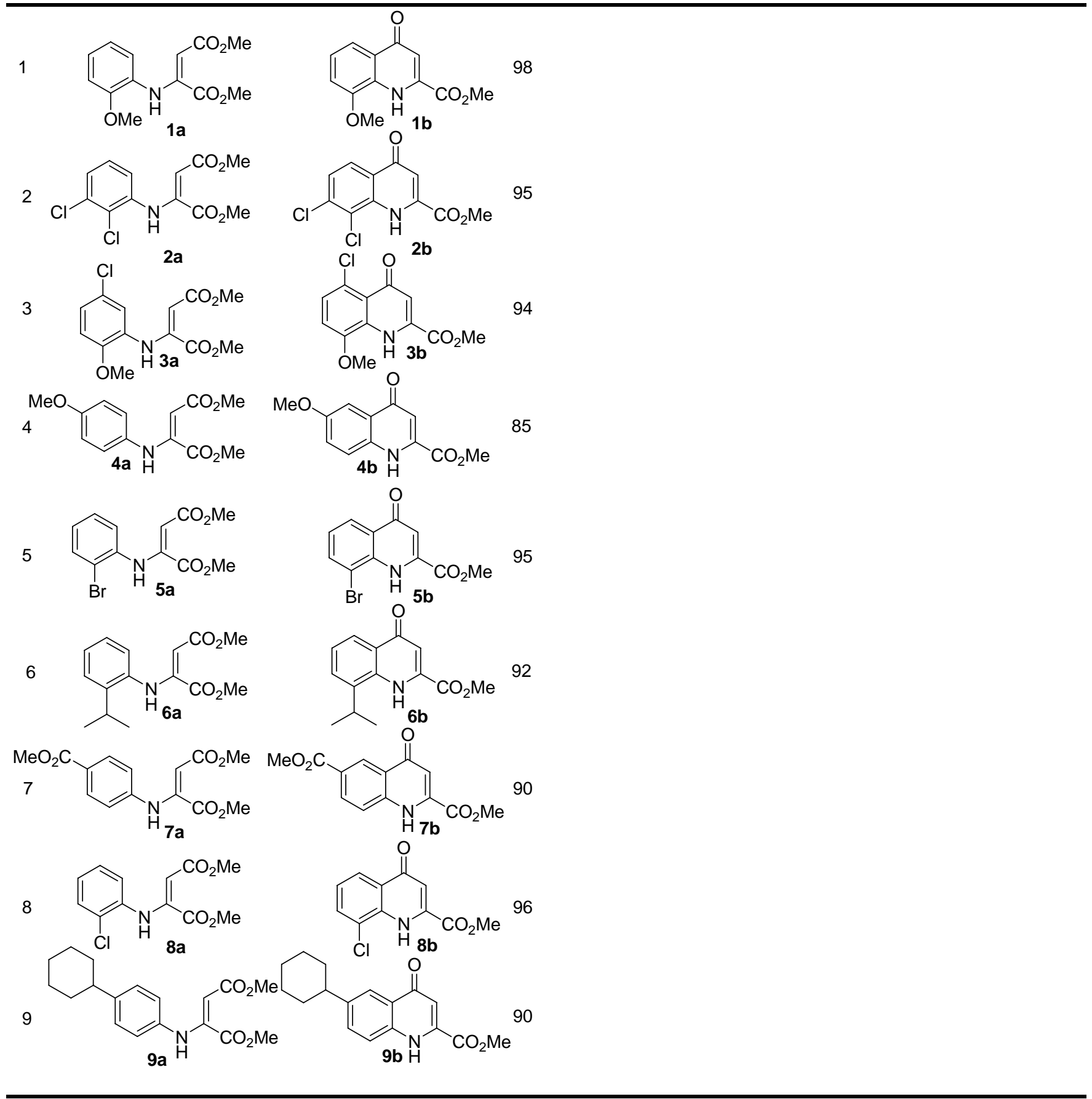

\section{Spectral Data for enamine substarates and 4-quinolone products (Table-1)}

1a: ${ }^{1} \mathrm{H}$ NMR (500 MHz, DMSO-d $)$ ): $\delta$ ppm $9.66(\mathrm{~s}, 1 \mathrm{H}), 7.06(\mathrm{~m}, 1 \mathrm{H}), 7.03(\mathrm{dd}, J=8.2,1.8 \mathrm{~Hz}, 1 \mathrm{H})$, $6.88(\mathrm{~m}, 1 \mathrm{H}), 6.79(\mathrm{~d}, J=8.2,1.8 \mathrm{~Hz}, 1 \mathrm{H}), 5.25(\mathrm{~s}, 1 \mathrm{H}), 3.80(\mathrm{~s}, 3 \mathrm{H}), 3.69(\mathrm{~s}, 3 \mathrm{H}), 3.67(\mathrm{~s}, 3 \mathrm{H}) .{ }^{13} \mathrm{C}$ 
NMR (125 MHz, DMSO-d $\left.{ }_{6}\right): \delta$ ppm 169.0, 163.82, 149.9, 147.7, 128.4, 124.5, 120.6, 119.6, 111.5, 91.1, 55.5, 52.8, 51.1. Yellow solid, m/z: 265.0950 [M+]. ESI-HRMS Calcd. for $\mathrm{C}_{13} \mathrm{H}_{15} \mathrm{NO}_{5}: 266.1028$ $[\mathrm{M}+\mathrm{H}]^{+}$. Found 266.1034 .

1b: ${ }^{1} \mathrm{H}$ NMR (400 MHz, $\left.\mathrm{D}_{3} \mathrm{CCO}_{2} \mathrm{D}\right): \delta$ ppm $7.86(\mathrm{~d}, J=8.2 \mathrm{~Hz}, 1 \mathrm{H}), 7.38(\mathrm{t}, J=8.2 \mathrm{~Hz}, 1 \mathrm{H}), 7.25$ (d, $J=8.2 \mathrm{~Hz}, 1 \mathrm{H}), 7.19(\mathrm{~s}, 1 \mathrm{H}), 4.07(\mathrm{~s}, 3 \mathrm{H}), 4.06(\mathrm{~s}, 3 \mathrm{H}) .{ }^{13} \mathrm{C} \mathrm{NMR}\left(100 \mathrm{MHz}, \mathrm{D}_{3} \mathrm{CCO}_{2} \mathrm{D}\right): \delta \mathrm{ppm} 181.3$, 163.5, 149.7, 137.9, 131.6, 127.1, 126.3, 117.7, 112.9, 111.4, 57.1, 54.6. White solid, m/z: 233.0688 $\left[\mathrm{M}^{+}\right]$. ESI-HRMS Calcd. for $\mathrm{C}_{12} \mathrm{H}_{11} \mathrm{NO}_{4} ; 256.0586[\mathrm{M}+\mathrm{Na}]^{+}$. Found 256.0586

2a: ${ }^{1} \mathrm{H} \mathrm{NMR}\left(400 \mathrm{MHz}, \mathrm{CDCl}_{3}\right): \delta \mathrm{ppm} 9.80(\mathrm{~s}, 1 \mathrm{H}), 7.17(\mathrm{dd}, J=8.0,1.0 \mathrm{~Hz}, 1 \mathrm{H}), 7.08(\mathrm{t}, J=8.0 \mathrm{~Hz}$, 1H), $6.66(\mathrm{dd}, J=8.0,1.0 \mathrm{~Hz}, 1 \mathrm{H}), 5.61(\mathrm{~s}, 1 \mathrm{H}), 3.78(\mathrm{~s}, 3 \mathrm{H}), 3.74(\mathrm{~s}, 3 \mathrm{H}) .{ }^{13} \mathrm{C} \mathrm{NMR}(100 \mathrm{MHz}$, $\left.\mathrm{CDCl}_{3}\right): \delta$ ppm 169.6, 164.4, 146.1, 139.4, 133.9, 127.2, 125.3, 124.3, 118.7, 97.6, 523.2, 51.8. Yellow solid, m/z: $303.0065\left[\mathrm{M}^{+}\right]$. ESI-HRMS Calcd. for $\mathrm{C}_{12} \mathrm{H}_{11} \mathrm{Cl}_{2} \mathrm{NO}_{4} ; 325.9963[\mathrm{M}+\mathrm{Na}]^{+}$. Found 325.9957.

2b: ${ }^{1} \mathrm{H}$ NMR (400 MHz, $\mathrm{CDCl}_{3}$ ): $\delta$ ppm 9.33 (s, 1H), $8.19(\mathrm{~d}, J=8.8 \mathrm{~Hz}, 1 \mathrm{H}), 7.44(\mathrm{~d}, J=8.8 \mathrm{~Hz}, 1 \mathrm{H})$, $6.98(\mathrm{~s}, 1 \mathrm{H}), 4.07(\mathrm{~s}, 3 \mathrm{H}) .{ }^{13} \mathrm{C} \mathrm{NMR}\left(100 \mathrm{MHz}, \mathrm{CDCl}_{3}\right): \delta \mathrm{ppm} 178.8,162.9,137.9,137.1,136.7$, 125.8, 125.8, 125.7, 120.9, 112.7, 54.3. Off-white solid, m/z: $270.9803\left[\mathrm{M}^{+}\right]$. ESI-HRMS Calcd. for $\mathrm{C}_{11} \mathrm{H}_{7} \mathrm{Cl}_{2} \mathrm{NO}_{3} ; 271.9881,[\mathrm{M}+\mathrm{H}]^{+}$. Found 271.9878

3a: ${ }^{1} \mathrm{H} \mathrm{NMR}\left(500 \mathrm{MHz}, \mathrm{CDCl}_{3}\right): \delta \mathrm{ppm} 9.63(\mathrm{~s}, 1 \mathrm{H}), 6.98(\mathrm{~d}, J=8.7,1.6 \mathrm{~Hz}, 1 \mathrm{H}), 6.78(\mathrm{~d}, J=8.7,1 \mathrm{H})$, $6.74(\mathrm{~s}, 1 \mathrm{H}), 5.47(\mathrm{~s}, 1 \mathrm{H}), 3.84(\mathrm{~s}, 3 \mathrm{H}), 3.77(\mathrm{~s}, 3 \mathrm{H}), 3.76(\mathrm{~s}, 3 \mathrm{H}) .{ }^{13} \mathrm{C} \mathrm{NMR}\left(125 \mathrm{MHz}, \mathrm{CDCl}_{3}\right): \delta \mathrm{ppm}$ 169.9, 164.6, 149.3, 147.1, 130.8, 125.7, 123.8, 120.1, 112.0, 94.6, 56.1, 52.9, 51.5. Yellow Solid, m/z: 299.0561 $\left[\mathrm{M}^{+}\right]$. ESI-HRMS Calcd. for $\mathrm{C}_{13} \mathrm{H}_{14} \mathrm{ClNO}_{5} ; 322.0458[\mathrm{M}+\mathrm{Na}]^{+}$. Found 322.0458 .

3b: ${ }^{1} \mathrm{H}$ NMR $\left(500 \mathrm{MHz}, \mathrm{CD}_{3} \mathrm{CO}_{2} \mathrm{D}\right): \delta \mathrm{ppm} 7.43(\mathrm{~d}, J=8.3 \mathrm{~Hz}, 1 \mathrm{H}), 7.33(\mathrm{~s}, 1 \mathrm{H}), 7.25(\mathrm{~d}, J=8.3 \mathrm{~Hz}$, 1H), $4.11(\mathrm{~s}, 3 \mathrm{H}), 4.09(\mathrm{~s}, 3 \mathrm{H})$. Note: DMSO at 2.86. ${ }^{13} \mathrm{C} \mathrm{NMR}\left(125 \mathrm{MHz}, \mathrm{CDCl}_{3}\right): \delta$ ppm 179.1, 162.4, 148.5, 138.2, 133.5, 129.4, 124.2, 121.9, 113.5, 112.3, 57.6, 54.9. Off-white solid, m/z: 267.0298 [M+ ${ }^{+}$. ESI-HRMS Calcd. for $\mathrm{C}_{12} \mathrm{H}_{10} \mathrm{ClNO}_{4}: 268.0377[\mathrm{M}+\mathrm{H}]^{+}$, found 268.0308.

4a: No satisfactory NMR data was obtained. Yellow solid, m/z: $265.095\left[\mathrm{M}^{+}\right]$. ESI-HRMS Calcd. for $\mathrm{C}_{13} \mathrm{H}_{15} \mathrm{NO}_{5}: 288.0848[\mathrm{M}+\mathrm{Na}]^{+}$, found 288.0851. 
4b: ${ }^{1} \mathrm{H}$ NMR (400 MHz, $\left.\mathrm{D}_{3} \mathrm{CCO}_{2} \mathrm{D}\right): \delta$ ppm $7.86(\mathrm{~d}, J=9.2 \mathrm{~Hz}, 1 \mathrm{H}), 7.72(\mathrm{~d}, J=2.8 \mathrm{~Hz}, 1 \mathrm{H}), 7.42$ (dd, $J=9.2,2.8 \mathrm{~Hz}, 1 \mathrm{H}), 7.23(\mathrm{~s}, 1 \mathrm{H}) 4.05(\mathrm{~s}, 3 \mathrm{H}), 3.93(\mathrm{~s}, 3 \mathrm{H}) .{ }^{13} \mathrm{C} \mathrm{NMR}\left(100 \mathrm{MHz}, \mathrm{D}_{3} \mathrm{CCO}_{2} \mathrm{D}\right): \delta \mathrm{ppm}$ $180.2,164.0,158.91,137.98,135.9,127.9,126.6,122.1,110.1,104.5,56.3,54.5$. Light-yellow solid, m/z: 233.0688 $\left[\mathrm{M}^{+}\right]$. ESI-HRMS Calcd. for $\mathrm{C}_{12} \mathrm{H}_{11} \mathrm{NO}_{4}: 234.0766[\mathrm{M}+\mathrm{H}]^{+}$. Found 234.0760.

5a: ${ }^{1} \mathrm{H}$ NMR $\left(500 \mathrm{MHz}, \mathrm{CDCl}_{3}\right): \delta$ ppm $9.75(\mathrm{~s}, 1 \mathrm{H}), 7.58(\mathrm{dd}, J=7.8,1.6 \mathrm{~Hz}, 1 \mathrm{H}), 7.20(\mathrm{td}, J=7.8$, $1.6 \mathrm{~Hz}, 1 \mathrm{H}), 6.95(\mathrm{td}, J=7.8,1.6 \mathrm{~Hz}, 1 \mathrm{H}), 6.77(\mathrm{dd}, J=7.8,1.6 \mathrm{~Hz}, 1 \mathrm{H}), 5.56(\mathrm{~s}, 1 \mathrm{H}), 3.78(\mathrm{~s}, 3 \mathrm{H})$, $3.72(\mathrm{~s}, 3 \mathrm{H}) .{ }^{13} \mathrm{C} \mathrm{NMR}\left(125 \mathrm{MHz}, \mathrm{CDCl}_{3}\right): \quad \delta \mathrm{ppm} 169.6,164.5,146.6,138.9,133.2,127.9,125.1$, 121.05, 115.8, 96.1, 52.9, 51.6. Light-yellow solid, m/z: $312.995\left[\mathrm{M}^{+}\right]$. ESI-HRMS Calcd. for $\mathrm{C}_{12} \mathrm{H}_{12} \mathrm{BrNO}_{4}: 314.0028[\mathrm{M}+\mathrm{H}]^{+}$, found 314.0027

5b: ${ }^{1} \mathrm{H}$ NMR (400 MHz, $\left.\mathrm{CDCl}_{3}\right): \delta$ ppm 9.39 (brs, $\left.1 \mathrm{H}\right), 8.30(\mathrm{dd}, J=8.0,1.2 \mathrm{~Hz}, 1 \mathrm{H}), 7.90(\mathrm{td}, J=8.0$, $1.2 \mathrm{~Hz}, 1 \mathrm{H}), 7.26(\mathrm{td}, J=8.0,1 \mathrm{H}), 6.99(\mathrm{~d}, J=1.2 \mathrm{~Hz}, 1 \mathrm{H}), 4.07(\mathrm{~s}, 3 \mathrm{H}) .{ }^{13} \mathrm{C} \mathrm{NMR}\left(100 \mathrm{MHz}, \mathrm{CDCl}_{3}\right)$ : $\delta$ ppm 179.1, 162.9, 126.9, 136.7, 136.3, 127.5, 126.2, 125.2, 112.4, 112.0, 54.2. White solid, m/z: $280.9688\left[\mathrm{M}^{+}\right]$. ESI-HRMS Calcd. for $\mathrm{C}_{11} \mathrm{H}_{8} \mathrm{BrNO}_{3}: 303.9579[\mathrm{M}+\mathrm{Na}]^{+}$. Found 303.9577.

6a: ${ }^{1} \mathrm{H}$ NMR (400 MHz, $\left.\mathrm{CDCl}_{3}\right): \delta$ ppm $9.65(\mathrm{~s}, 1 \mathrm{H}), 7.29(\mathrm{dd} J=7.6,1.6 \mathrm{~Hz}, 1 \mathrm{H}), 7.13(\mathrm{td}, J=7.6,1.6$ Hz, 1H), 7.08 (td, J=7.6, 1.6 Hz, 1H), 6.76(dd, J=7.6, 1.6 Hz, 1H), $5.40(\mathrm{~s}, 1 \mathrm{H}), 3.74(\mathrm{~s}, 3 \mathrm{H}), 3.61(\mathrm{~s}$, 3H), 3.27 (septet, $J=6.8 \mathrm{~Hz}, 1 \mathrm{H}), 1.28(\mathrm{~d}, J=6.8 \mathrm{~Hz}, 6 \mathrm{H}) .{ }^{13} \mathrm{C} \mathrm{NMR}\left(100 \mathrm{MHz}, \mathrm{CDCl}_{3}\right): \delta \mathrm{ppm} 170.5$, $164.8,149.7,141.4,138.0,126.3,126.1,125.7,122.7,92.5,52.6,51.2,28.4,22.9$. Yellow oil, m/z: 277.1314 [M+ $\mathrm{M}^{+}$. ESI-HRMS Calcd. for $\mathrm{C}_{15} \mathrm{H}_{19} \mathrm{NO}_{4}: 278.1392[\mathrm{M}+\mathrm{H}]^{+}$, found 278.1393.

6b: ${ }^{1} \mathrm{H}$ NMR $\left(400 \mathrm{MHz}, \mathrm{CDCl}_{3}\right): \delta$ ppm $9.09(\mathrm{~s}, 1 \mathrm{H}), 8.23(\mathrm{~d}, J=7.8 \mathrm{~Hz}, 1 \mathrm{H}), 7.59(\mathrm{~d}, J=7.8 \mathrm{~Hz}, 1 \mathrm{H})$, 7.35(t, $J=7.8 \mathrm{~Hz}, 1 \mathrm{H}), 6.95(\mathrm{~d}, J=1.6 \mathrm{~Hz}, 1 \mathrm{H}), 4.05(\mathrm{~s}, 3 \mathrm{H}), 3.28$ (septet, $J=6.8 \mathrm{~Hz}, 1 \mathrm{H}), 1.42(\mathrm{~d}, J=$ $6.8 \mathrm{~Hz}, 6 \mathrm{H}) .{ }^{13} \mathrm{C} \mathrm{NMR}\left(100 \mathrm{MHz}, \mathrm{CDCl}_{3}\right): \delta \mathrm{ppm} 180.2,163.9,136.6,135.8,135.8,129.3,126.9$, 124.5, 124.3, 111.3, 54.0, 27.8, 22.9. Light-yellow solid, m/z: 245.1052 [M $\left.{ }^{+}\right]$. ESI-HRMS Calcd. for $\mathrm{C}_{14} \mathrm{H}_{15} \mathrm{NO}_{3}: 268.0950[\mathrm{M}+\mathrm{Na}]^{+}$, found 268.0944.

7a: ${ }^{1} \mathrm{H}$ NMR (400 MHz, $\left.\mathrm{CDCl}_{3}\right): \delta \mathrm{ppm} 9.72(\mathrm{~s}, 1 \mathrm{H}), 7.94(\mathrm{~m}, 2 \mathrm{H}), 6.85(\mathrm{~m}, 2 \mathrm{H}), 5.53(\mathrm{~s}, 1 \mathrm{H}), 3.87(\mathrm{~s}$, 3H), $3.74(\mathrm{~s}, 3 \mathrm{H}), 3.72(\mathrm{~s}, 3 \mathrm{H}) .{ }^{13} \mathrm{C} \mathrm{NMR}\left(100 \mathrm{MHz}, \mathrm{CDCl}_{3}\right): \delta \mathrm{ppm} 169.6,166.7,164.6,146.5,144.6$, 
131.3, 131.0, 125.3, 120.5, 119.3, 96.9, 53.1, 52.1, 51.6. Yellow solid, m/z: 293.0899 [M ${ }^{+}$. ESI-

HRMS Calcd. for $\mathrm{C}_{14} \mathrm{H}_{15} \mathrm{NO}_{6}: 294.0978[\mathrm{M}+\mathrm{H}]^{+}$, found 294.09

7b: ${ }^{1} \mathrm{H}$ NMR (400 MHz, $\left.\mathrm{CF}_{3} \mathrm{CO}_{2} \mathrm{D}\right): \delta$ ppm $9.34(\mathrm{~m}, 1 \mathrm{H}), 8.78(\mathrm{~m}, 1 \mathrm{H}), 8.43(\mathrm{~m}, 1 \mathrm{H}), 8.05(\mathrm{~m}, 1 \mathrm{H}) 4.26$

(m, 3H), $4.17(\mathrm{~m}, 3 \mathrm{H}) .{ }^{13} \mathrm{C} \mathrm{NMR}\left(100 \mathrm{MHz}, \mathrm{CF}_{3} \mathrm{CO}_{2} \mathrm{D}\right): \delta \mathrm{ppm} 175.8,170.0,162.9,144.9,144.1$, 138.9, 133.3, 129.6, 123.5, 123.2, 109.3, 57.6, 55.9. White solid, m/z: 261.0637 [M $\left.{ }^{+}\right]$. ESI-HRMS Calcd. for $\mathrm{C}_{13} \mathrm{H}_{11} \mathrm{NO}_{5}: 262.0715[\mathrm{M}+\mathrm{H}]^{+}$, found 262.0721 .

8a: ${ }^{1} \mathrm{H}$ NMR (400 MHz, $\left.\mathrm{CDCl}_{3}\right): \delta$ ppm $9.78(\mathrm{~s}, 1 \mathrm{H}), 7.39(\mathrm{dd}, J=8.0,1.6 \mathrm{~Hz}, 1 \mathrm{H}), 7.15(\mathrm{td}, J=8.0,1.6$ $\mathrm{Hz}, 1 \mathrm{H}), 7.01(\mathrm{td}, J=8.0,1.6 \mathrm{~Hz}, 1 \mathrm{H}), 6.78(\mathrm{dd}, J=8.0,1.6 \mathrm{~Hz}, 1 \mathrm{H}), 5.55(\mathrm{~s}, 1 \mathrm{H}), 3.77(\mathrm{~s}, 3 \mathrm{H}), 3.72(\mathrm{~s}$, $3 \mathrm{H}) .{ }^{13} \mathrm{C} \mathrm{NMR}\left(100 \mathrm{MHz}, \mathrm{CDCl}_{3}\right): \delta \mathrm{ppm} 169.7,164.6,146.7,137.6,130.1,127.3,125.7,124.7,121.0$, 96.1, 53.0, 51.6. Yellow solid, m/z: 269.0455 [M+ $\mathrm{M}^{+}$. ESI-HRMS Calcd. for $\mathrm{C}_{12} \mathrm{H}_{12} \mathrm{ClNO}_{4}: 270.0533$ $[\mathrm{M}+\mathrm{H}]^{+}$, found 270.0530 .

8b: ${ }^{1} \mathrm{H}$ NMR $\left(400 \mathrm{MHz}, \mathrm{CDCl}_{3}\right): \delta$ ppm 9.13 (very br s, $\left.1 \mathrm{H}\right), 8.22(\mathrm{dd}, J=8.0,1.2 \mathrm{~Hz}, 1 \mathrm{H}), 7.72(\mathrm{dd}, J=$ 7.8, $1.2 \mathrm{~Hz}, 1 \mathrm{H}), 7.31(\mathrm{t}, J=8.0 \mathrm{~Hz}, 1 \mathrm{H}), 6.93(\mathrm{~s}, 1 \mathrm{H}), 4.07(\mathrm{~s}, 3 \mathrm{H}) .{ }^{13} \mathrm{C} \mathrm{NMR}\left(100 \mathrm{MHz}, \mathrm{CDCl}_{3}\right): \delta \mathrm{ppm}$ $178.9,162.9,136.4,135.9,132.7,127.4,125.3,124.5,122.5,112.1,54.1$. White solid, m/z: 237.0193 $\left[\mathrm{M}^{+}\right]$. ESI-HRMS Calcd. for $\mathrm{C}_{11} \mathrm{H}_{8} \mathrm{ClNO}_{3}: 260.0090[\mathrm{M}+\mathrm{Na}]^{+}$, found 260.0084 .

9a: No satisfactory NMR and ESI-HRMS data was obtained

9b: ${ }^{1} \mathrm{H}$ NMR (400 MHz, CD $\left.3 \mathrm{OD}\right): \delta$ ppm $8.09(\mathrm{~d}, J=2.0 \mathrm{~Hz}, 1 \mathrm{H}), 7.81(\mathrm{~d}, J=8.8 \mathrm{~Hz}, 1 \mathrm{H}), 7.70(\mathrm{dd}, J=$ 8.8, 2.0 Hz, 1H), $6.98(\mathrm{~s}, 1 \mathrm{H}), 4.04(\mathrm{~s}, 3 \mathrm{H}), 2.69(\mathrm{~m}, 1 \mathrm{H}), 1.91(\mathrm{~m}, 4 \mathrm{H}), 1.79(\mathrm{~m}, 1 \mathrm{H}), 1.58-1.43$ (om, 4H), $1.33(\mathrm{~m}, 1 \mathrm{H})$. Note; DMSO at $2.70 .{ }^{13} \mathrm{C} \mathrm{NMR}\left(100 \mathrm{MHz}, \mathrm{CD}_{3} \mathrm{OD}\right): \delta \mathrm{ppm} 180.8,163.7,147.1$, 140.2, 140.1, 134.8, 126.9, 122.8, 120.7, 110.6, 54.3, 45.8, 35.7, 28.0, 27.3. Light-yellow solid, m/z: 285.1365 [M+ ${ }^{+}$. ESI-HRMS Calcd. for $\mathrm{C}_{17} \mathrm{H}_{19} \mathrm{NO}_{3}: 308.1263[\mathrm{M}+\mathrm{Na}]^{+}$, found 308.1259 .

\section{General Procedure for preparation of enamine diester adducts. (Table 2, 10a - 12a):}

To a solution of $o$-chloroaniline $(2 \mathrm{~g}, 16.3 \mathrm{mmol})$ in toluene $(10 \mathrm{~mL})$ was added dimethyl methoxymethylenemalonate $(3.4 \mathrm{~g}, 19.6 \mathrm{mmol}, 1.2 \mathrm{eq})$. The mixture was aged at reflux $\left(110^{\circ} \mathrm{C}\right)$ over 6 h. Reaction mixture was cooled to $\mathrm{rt}$ and MTBE $(20 \mathrm{~mL})$ was added. Product is filtered and cake washed with heptane. After overnight drying in vac oven at $50{ }^{\circ} \mathrm{C}, 4.0 \mathrm{~g}$ of $10 \mathrm{a}(15.1 \mathrm{mmol}, 93 \%)$ was 
collected in $96 \mathrm{wt} \%$. Crude solid was directly used for the subsequent cyclization reaction. This procedure was applied to the preparation of bis-enamine adducts (Table 3, 13a - 16a) and heterocyclic enamines; 19a, 21a, 24a, and 25a.

Table 2: Synthesis of 3-Carboxy-4-Quinolones

Entry Reactant Product \% Yield

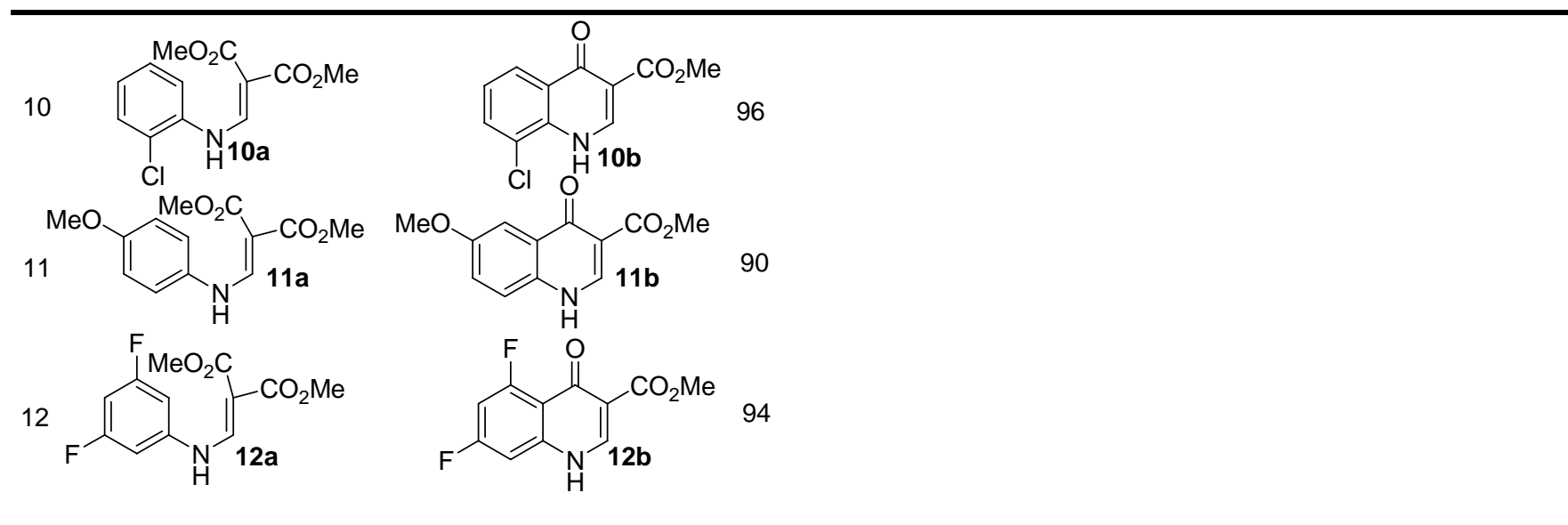

\section{General Procedure for the preparation of 4-quinolones (10b - 12b).}

Enamine 10 a (1.g, $3.8 \mathrm{mmol})$, was added to a RBF that was equipped with a thermocouple and a stirrer, Eaton's reagent $(4 \mathrm{~mL})$ was then added to the flask. Reaction mixture was aged at $90{ }^{\circ} \mathrm{C}$, and progress of reaction was followed by HPLC. When conversion was $\geq 98 \%$, reaction mixture was cooled to $5{ }^{\circ} \mathrm{C}$ and slowly transferred to an excess saturated sodium carbonate solution (10 eq) that was cooled to 10 ${ }^{\circ} \mathrm{C}$. The solid was filtered, washed with water $(10 \mathrm{~mL})$ and was dried in a vac. oven at $50{ }^{\circ} \mathrm{C}$ over $12 \mathrm{~h}$. $0.9 \mathrm{~g}$ of 10b $(3.7 \mathrm{mmol}, 97 \%)$ was collected in $98 \mathrm{wt} \%$ purity. The same cyclization protocol was applied to the preparation of heterocyclic quinolones; 19b, 21b, 24b, and 25b.

\section{Spectral Data for enamine substrates and 4-quinolone products (Table- 2)}

10a: ${ }^{1} \mathrm{H}$ NMR (400 MHz, $\left.\mathrm{CD}_{3} \mathrm{CN}\right): \delta$ ppm 11.28 (d, J=12.8 Hz, 1H), 8.55 (d, J=12.8 Hz, 1H), 7.47 $(\mathrm{ddd}, J=9.4,8.0,1.2 \mathrm{~Hz}, 1 \mathrm{H}), 7.37(\mathrm{~m}, 1 \mathrm{H}), 7.13(\mathrm{td}, J=7.8,1.8 \mathrm{~Hz}, 1 \mathrm{H}), 3.79(\mathrm{~s}, 3 \mathrm{H}), 3.73(\mathrm{~s}, 3 \mathrm{H}) .{ }^{13} \mathrm{C}$ NMR (100 MHz, $\left.\mathrm{CD}_{3} \mathrm{CN}\right): \delta$ ppm 169.8, 166.3, 152.0, 137.3, 131.1, 129.6, 126.3, 124.0, 117.3, 95.7, 52.2, 51.9. White solid, m/z: $269.0455\left[\mathrm{M}^{+}\right]$. ESI-HRMS Calcd. for $\mathrm{C}_{12} \mathrm{H}_{12} \mathrm{ClNO}_{4}: 292.0353[\mathrm{M}+\mathrm{Na}]^{+}$, found 292.0356 . 
10b: ${ }^{1} \mathrm{H}$ NMR $\left(400 \mathrm{MHz}, \mathrm{F}_{3} \mathrm{CCO}_{2} \mathrm{D}\right): \delta \mathrm{ppm} 9.37(\mathrm{~s}, 1 \mathrm{H}), 8.60(\mathrm{~d}, J=7.6 \mathrm{~Hz}, 1 \mathrm{H}), 8.26(\mathrm{~d}, J=7.6 \mathrm{~Hz}$ 1H), $7.91(\mathrm{t}, J=7.6 \mathrm{~Hz}, 1 \mathrm{H}), 4.18(\mathrm{~s}, 3 \mathrm{H}) .{ }^{13} \mathrm{C} \mathrm{NMR}\left(100 \mathrm{MHz}, \mathrm{F}_{3} \mathrm{CCO}_{2} \mathrm{D}\right): \delta \mathrm{ppm} \mathrm{176.4,} \mathrm{169.9,} \mathrm{148.4,}$ 139.8, 138.4, 132.7, 127.6, 126.11, 123.8, 107.81, 56.1. Brown solid, m/z: 237.0193 [M ${ }^{+}$. ESI-HRMS Calcd. for $\mathrm{C}_{11} \mathrm{H}_{8} \mathrm{ClNO}_{3}: 238.0271[\mathrm{M}+\mathrm{H}]^{+}$, found 238.0268.

11a: ${ }^{1} \mathrm{H}$ NMR (400 MHz, $\left.\mathrm{CDCl}_{3}\right): \delta$ ppm $10.97(\mathrm{~d}, J=14.0 \mathrm{~Hz}, 1 \mathrm{H}), 8.41(\mathrm{~d}, J=14.0 \mathrm{~Hz}, 1 \mathrm{H}), 7.05$ (m, 2H), $6.87(\mathrm{~m}, 2 \mathrm{H}), 3.82(\mathrm{~s}, 3 \mathrm{H}), 3.77(\mathrm{~s}, 3 \mathrm{H}), 3.74(\mathrm{~s}, 3 \mathrm{H}) .{ }^{13} \mathrm{C} \mathrm{NMR}\left(100 \mathrm{MHz}, \mathrm{CDCl}_{3}\right): \delta \mathrm{ppm} 169.6$, 166.2, 157.4, 153.0, 132.7, 119.0, 115.1, 91.9, 55.7, 51.5, 51.4. White solid, m/z: 265.0950 [M ${ }^{+}$. ESIHRMS Calcd. for $\mathrm{C}_{13} \mathrm{H}_{15} \mathrm{NO}_{5}: 288.0848[\mathrm{M}+\mathrm{Na}]^{+}$, found 288.0850.

11b: ${ }^{1} \mathrm{H}$ NMR $\left(400 \mathrm{MHz}, \mathrm{CF}_{3} \mathrm{CO}_{2} \mathrm{D}\right): \delta$ ppm $9.11(\mathrm{~s}, 1 \mathrm{H}), 8.02(\mathrm{~d}, J=9.2 \mathrm{~Hz}, 1 \mathrm{H}), 7.89(\mathrm{~s}, 1 \mathrm{H}), 7.82(\mathrm{~d}$, $J=9.2 \mathrm{~Hz}, 1 \mathrm{H}), 4.17(\mathrm{~s}, 3 \mathrm{H}), 4.06(\mathrm{~s}, 3 \mathrm{H}) .{ }^{13} \mathrm{C} \mathrm{NMR}\left(100 \mathrm{MHz}, \mathrm{CF}_{3} \mathrm{CO}_{2} \mathrm{D}\right): \delta \mathrm{ppm} 174.2,170.5,144.4$, 137.2, 132.6, 124.3, 123.9, 106.9, 104.8, 57.7, 55.8. White solid, m/z: 233.0688 [M $\left.{ }^{+}\right]$. ESI-HRMS Calcd. for $\mathrm{C}_{12} \mathrm{H}_{11} \mathrm{NO}_{4}: 234.0766[\mathrm{M}+\mathrm{H}]^{+}$, found 234.0762 .

12a: ${ }^{1} \mathrm{H}$ NMR (400 MHz, $\left.\mathrm{CDCl}_{3}\right): \delta \mathrm{ppm} 10.98(\mathrm{~d}, J=13.2 \mathrm{~Hz}, 1 \mathrm{H}), 8.38(\mathrm{~d}, J=13.2 \mathrm{~Hz}, 1 \mathrm{H}), 6.66(\mathrm{~m}$, 2H), $6.57(\mathrm{tt}, J=8.8,2.2 \mathrm{~Hz}, 1 \mathrm{H}), 3.83(\mathrm{~s}, 3 \mathrm{H}), 3.77(\mathrm{~s}, 3 \mathrm{H}) .{ }^{13} \mathrm{C} \mathrm{NMR}\left(100 \mathrm{MHz}, \mathrm{CDCl}_{3}\right): \delta \mathrm{ppm}$ 169.1. $\left(\mathrm{dd}, J_{C F}=241.9,15.2 \mathrm{~Hz}\right), 151.2141 .7\left(\mathrm{t}, J_{C F}=12.4 \mathrm{~Hz}\right), 100.58(\mathrm{~m}), 100.2\left(\mathrm{t}, J_{C F}=25.2 \mathrm{~Hz}\right)$, 95.1, 51.9, 51.8. Off-white solid, m/z: $271.0656\left[\mathrm{M}^{+}\right]$. ESI-HRMS Calcd. for $\mathrm{C}_{12} \mathrm{H}_{11} \mathrm{~F}_{2} \mathrm{NO}_{4}: 272.0734$ $[\mathrm{M}+\mathrm{H}]^{+}$, found 272.0737 .

12b: ${ }^{1} \mathrm{H}$ NMR (400 MHz, $\left.\mathrm{CF}_{3} \mathrm{CO}_{2} \mathrm{D}\right): \delta$ ppm $9.25(\mathrm{~s}, 1 \mathrm{H}), 7.60(\mathrm{dd}, J=8.0,2.0 \mathrm{~Hz}, 1 \mathrm{H}), 7.31(\mathrm{td}, J=8.8$, $2.0 \mathrm{~Hz}, 1 \mathrm{H}), 4.13(3,3 \mathrm{H}) .{ }^{13} \mathrm{C} \mathrm{NMR}\left(100 \mathrm{MHz}, \mathrm{CF}_{3} \mathrm{CO}_{2} \mathrm{D}\right): \delta \mathrm{ppm} 176.3\left(\mathrm{~d}, J_{C F}=2.9 \mathrm{~Hz}\right), 170.7(\mathrm{dd}$, $\left.J_{C F}=266.8,13.3 \mathrm{~Hz}\right), 170.2,164.5\left(\mathrm{dd}, J_{C F}=275.7,15.0 \mathrm{~Hz}\right), 149.3,144.2\left(\mathrm{dd}, J_{C F}=15.1,4.5 \mathrm{~Hz}\right)$, $110.8\left(\mathrm{dd}, J_{C F}=13.6,2.0 \mathrm{~Hz}\right), 109.2\left(\mathrm{dd}, J_{C F}=27.9,23.6 \mathrm{~Hz}\right), 107.3,104.7\left(\mathrm{dd}, J_{C F}=26.3,4.9 \mathrm{~Hz}\right), 56.0$. White solid, m/z: 239.0394 $\left[\mathrm{M}^{+}\right]$. ESI-HRMS Calcd. for $\mathrm{C}_{11} \mathrm{H}_{7} \mathrm{~F}_{2} \mathrm{NO}_{3}: 240.0472[\mathrm{M}+\mathrm{H}]^{+}$, found 240.0473.

7. General Procedure for preparation of bis-enamine tetra ester adducts (Table 3, 13a - 16a). Refer to procedure 1.

8. General Procedure for preparation of bis-quinolones (13b-16b). Refer to procedure 2 . 
Table 3: Bis-Cyclization of Diamine Derivatives

Entry Reactant $\quad$ Product $\%$ Yield

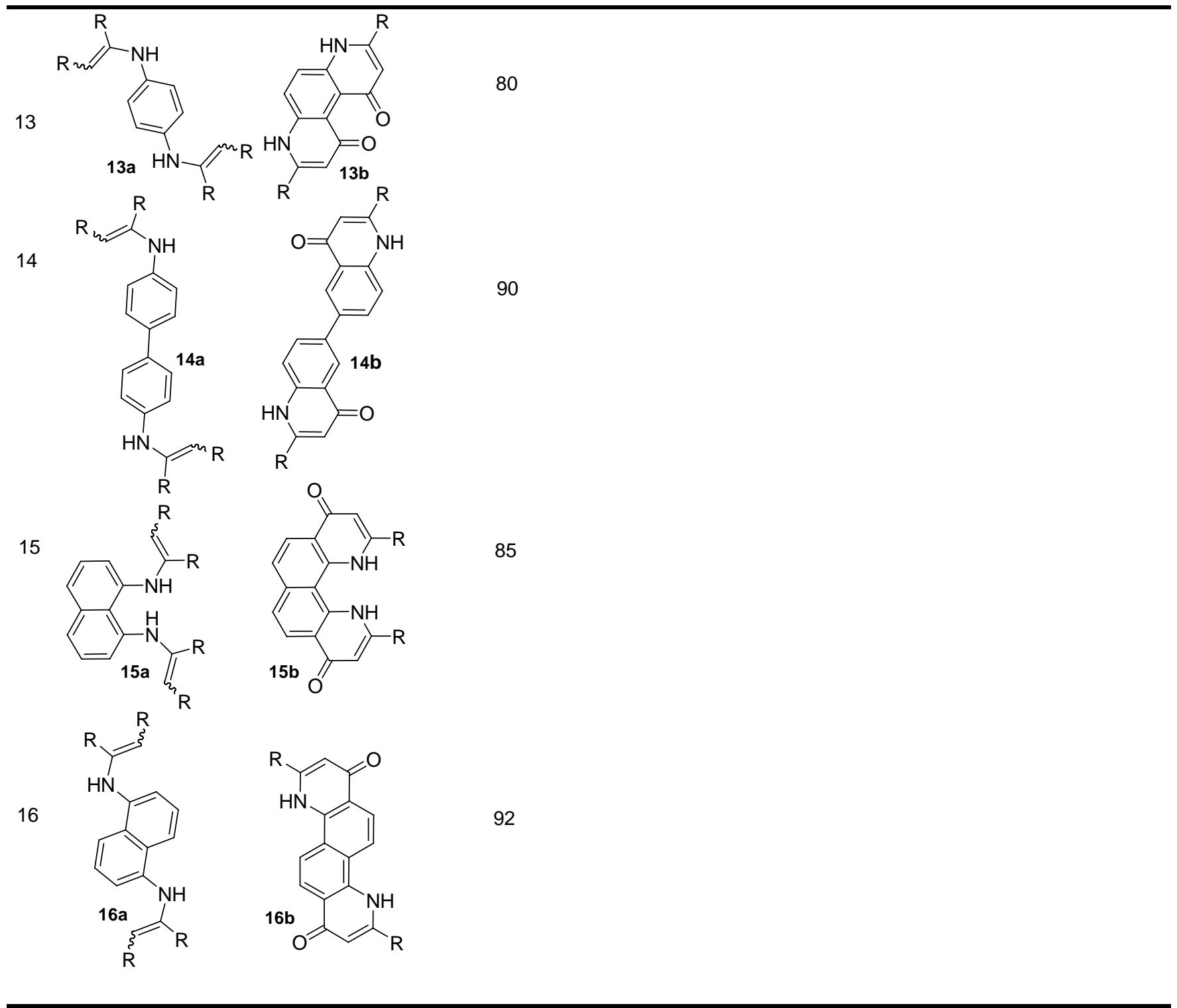

\section{Spectral Data for bis-enamine substrates and bis-quinolone products (Table-3):}

13a: ${ }^{1} \mathrm{H}$ NMR $\left(400 \mathrm{MHz}, \mathrm{CDCl}_{3}\right): \delta$ ppm $9.61(\mathrm{~s}, 2 \mathrm{H}), 6.83(\mathrm{~s}, 4 \mathrm{H}), 5.38(\mathrm{~s}, 2 \mathrm{H}), 3.73(\mathrm{~s}, 6 \mathrm{H}), 3.68(\mathrm{~s}$, $6 \mathrm{H}) .{ }^{13} \mathrm{C}$ NMR $\left(100 \mathrm{MHz}, \mathrm{CDCl}_{3}\right): \delta \mathrm{ppm} 170.1,164.9,148.2,137.1,121.9,93.7,52.9,51.4$. Yellow solid, m/z: $392.122\left[\mathrm{M}^{+}\right]$. ESI-HRMS Calcd. for $\mathrm{C}_{18} \mathrm{H}_{20} \mathrm{~N}_{2} \mathrm{O}_{8}: 393.1298[\mathrm{M}+\mathrm{H}]^{+}$, found 393.1295

13b: ${ }^{1} \mathrm{H}$ NMR (400 MHz, $\left.\mathrm{D}_{3} \mathrm{CCO}_{2} \mathrm{D}\right): \delta \mathrm{ppm} 8.51(\mathrm{~s}, 2 \mathrm{H}), 7.55(\mathrm{~s}, 2 \mathrm{H}), 4.09(\mathrm{~s}, 6 \mathrm{H})$. Note: DMSO at 2.84. ${ }^{13} \mathrm{C}$ NMR $\left(100 \mathrm{MHz}, \mathrm{D}_{3} \mathrm{CCO}_{2} \mathrm{D}\right): \delta \mathrm{ppm} 177.6,162.6,144.3,141.5,130.43,121.1,114.7$, 54.6. Yellow solid, m/z: $328.0695\left[\mathrm{M}^{+}\right]$. ESI-HRMS Calcd. for $\mathrm{C}_{16} \mathrm{H}_{12} \mathrm{~N}_{2} \mathrm{O}_{6}: 329.0774[\mathrm{M}+\mathrm{H}]^{+}$, found 329.0774 . 
14a: ${ }^{1} \mathrm{H}$ NMR $\left(400 \mathrm{MHz}, \mathrm{CDCl}_{3}\right): \delta \mathrm{ppm} 7.49(\mathrm{~m}, 4 \mathrm{H}), 6.95(\mathrm{~m}, 4 \mathrm{H}), 5.43(\mathrm{~s}, 2 \mathrm{H}), 3.76(\mathrm{~s}, 6 \mathrm{H}), 3.74(\mathrm{~s}$

$6 \mathrm{H}) .{ }^{13} \mathrm{C} \mathrm{NMR}\left(100 \mathrm{MHz}, \mathrm{CDCl}_{3}\right): \delta \mathrm{ppm} 170.1,165.1,147.9,139.6,136.3,127.6,121.09,9.17,53.1$, 51.5. Yellow solid, $\mathrm{m} / \mathrm{z}$ : $468.1533\left[\mathrm{M}^{+}\right]$. ESI-HRMS Calcd. for $\mathrm{C}_{24} \mathrm{H}_{24} \mathrm{~N}_{2} \mathrm{O}_{8}$ : $469.1611[\mathrm{M}+\mathrm{H}]^{+}$, found 469.1611.

14b: ${ }^{1} \mathrm{H}$ NMR (500 MHz, $\left.\mathrm{CF}_{3} \mathrm{CO}_{2} \mathrm{D}\right): \delta \mathrm{ppm} 9.04(\mathrm{~s}, 2 \mathrm{H}), 8.67(\mathrm{~m}, 2 \mathrm{H}), 8.61(\mathrm{~m}, 2 \mathrm{H}), 8.13(\mathrm{~s}, 2 \mathrm{H}), 4.35$ (s, 6H). $\quad{ }^{13} \mathrm{C}$ NMR $\left(125 \mathrm{MHz}, \mathrm{CF}_{3} \mathrm{CO}_{2} \mathrm{D}\right): \delta \mathrm{ppm} 174.6,163.1,143.6,142.7,141.9,138.6,124.9$, 124.0, 124.0, 108.9, 57.4. Yellow solid: $\mathrm{m} / \mathrm{z}$ : $404.1008\left[\mathrm{M}^{+}\right]$. ESI-HRMS Calcd. for $\mathrm{C}_{22} \mathrm{H}_{16} \mathrm{~N}_{2} \mathrm{O}_{6}$ : 405.1087 $[\mathrm{M}+\mathrm{H}]^{+}$, found 405.1088 .

15a: ${ }^{1} \mathrm{H}$ NMR (400 MHz, $\left.\mathrm{CDCl}_{3}\right): \delta \mathrm{ppm} 10.13(\mathrm{~s}, 2 \mathrm{H}), 7.65(\mathrm{~d}, J=7.8 \mathrm{~Hz}, 2 \mathrm{H}), 7.35(\mathrm{t}, J=7.8 \mathrm{~Hz}, 2 \mathrm{H})$, $6.93(\mathrm{~d}, J=7.8 \mathrm{~Hz}, 2 \mathrm{H}), 5.49(\mathrm{~s}, 2 \mathrm{H}), 3.68(\mathrm{~s}, 6 \mathrm{H}), 3.55(\mathrm{~s}, 6 \mathrm{H}) .{ }^{13} \mathrm{C} \mathrm{NMR}\left(100 \mathrm{MHz}, \mathrm{CDCl}_{3}\right): \delta \mathrm{ppm}$ 169.7, 165.1, 148.1, 137.2, 136.3, 126.6, 125.9, 123.5, 121.8, 93.9, 52.8, 51.2. Yellow solid, no satisfactory ESI-HRMS data was obtained.

15b: ${ }^{1} \mathrm{H}$ NMR (400 MHz, $\left.\mathrm{CF}_{3} \mathrm{CO}_{2} \mathrm{D}\right): \delta \mathrm{ppm} 8.77$ (d, $\left.J=8.8 \mathrm{~Hz}, 2 \mathrm{H}\right), 8.31(\mathrm{~d}, J=8.8 \mathrm{~Hz}, 2 \mathrm{H}), 8.15$ (s, 2H), 4.39 (s, 6H). Note: DMSO at 3.20. $\left.{ }^{13} \mathrm{C} \mathrm{NMR} \mathrm{(100} \mathrm{MHz,} \mathrm{CF}_{3} \mathrm{CO}_{2} \mathrm{D}\right): \delta \mathrm{ppm} 168.9,166.70,146.8$, 146.3, 142.3, 132.2, 126.8, 123.4, 118.7, 56.6. Note: DMSO at 40.96. Brown solid, m/z: $378.0852\left[\mathrm{M}^{+}\right]$. ESI-HRMS Calcd. for $\mathrm{C}_{20} \mathrm{H}_{14} \mathrm{~N}_{2} \mathrm{O}_{6}: 379.0930[\mathrm{M}+\mathrm{H}]^{+}$, found 379.0929.

16a: ${ }^{1} \mathrm{H}$ NMR (400 MHz, $\mathrm{CDCl}_{3}$ ): $\delta$ ppm 10.08 (br s, $\left.2 \mathrm{H}\right), 7.95$ (d, J=8.4 Hz, $\left.2 \mathrm{H}\right), 7.43$ (t, J=8.0 Hz, 2 H), $6.97(\mathrm{~d}, J=7.6 \mathrm{~Hz}, 2 \mathrm{H}), 5.56(\mathrm{~s}, 2 \mathrm{H}), 3.79(\mathrm{~s}, 6 \mathrm{H}), 3.61(\mathrm{~s}, 6 \mathrm{H}) .{ }^{13} \mathrm{C} \mathrm{NMR}\left(100 \mathrm{MHz}, \mathrm{CDCl}_{3}\right): \delta$ ppm 170.5, 164.9, 149.3, 137.3, 128.9, 126.1, 119.4, 118.8, 94.7, 52.9, 51.6. Yellow solid, m/z: 442.1376 [M ${ }^{+}$. ESI-HRMS Calcd. for $\mathrm{C}_{22} \mathrm{H}_{22} \mathrm{~N}_{2} \mathrm{O}_{8}: 443.1454[\mathrm{M}+\mathrm{H}]^{+}$, found 443.1454 .

16b: ${ }^{1} \mathrm{H}$ NMR (400 MHz, $\left.\mathrm{CF}_{3} \mathrm{CO}_{2} \mathrm{D}\right): \delta$ ppm 9.32 (d, J=8.8 Hz, 2H), 8.97 (d, J=8.8 Hz, 2H), 8.34 (s, 2H), $4.32(\mathrm{~s}, 6 \mathrm{H}) .{ }^{13} \mathrm{C}$ NMR (100 MHz, $\left.\mathrm{CF}_{3} \mathrm{CO}_{2} \mathrm{D}\right): \delta \mathrm{ppm} 175.0,162.2,143.9,139.4,128.5,125.4$, 125.0, 124.5, 111.5, 57.4. Light-brown solid, m/z: $378.0852\left[\mathrm{M}^{+}\right]$. ESI-HRMS Calcd. for $\mathrm{C}_{20} \mathrm{H}_{14} \mathrm{~N}_{2} \mathrm{O}_{6}$ : $379.0930[\mathrm{M}+\mathrm{H}]^{+}$, found 379.0925 .

10. General Procedure for preparation of heterocyclic enamine adducts (Table 4, 17c, 17a - 26a). Refer to procedures 1 and 4. 
11. General Procedure for preparation of heterocyclic quinolones (Table 4, 17d, 17b - 26b).

Refer to procedures 2 and 5.

Table 4: Synthesis of Heterocyclic Quinolones

Entry Reactant Product \% Yield

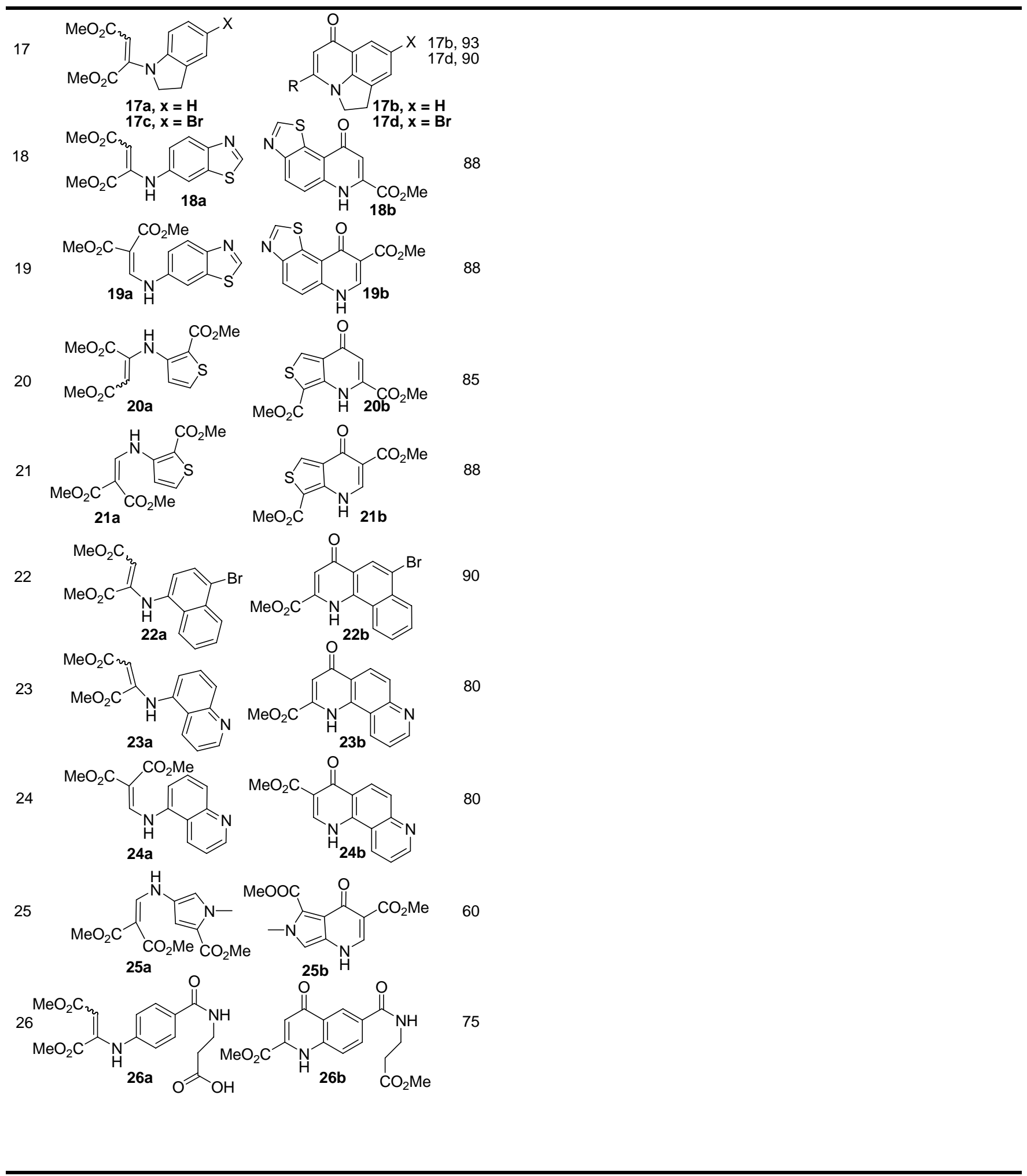




\section{Spectral Data for heterocyclic enamines and heterocyclic quinolones (Table-4):}

17a: ${ }^{1} \mathrm{H}$ NMR $\left(400 \mathrm{MHz}, \mathrm{CDCl}_{3}\right): \delta \mathrm{ppm} 7.18(\mathrm{~m}, 1 \mathrm{H}), 7.10(\mathrm{~m}, 1 \mathrm{H}), 6.94(\mathrm{td}, J=7.8,1.0 \mathrm{~Hz}, 1 \mathrm{H}), 6.83$ $(\mathrm{d}, J=7.8 \mathrm{~Hz}, 1 \mathrm{H}), 5.04(\mathrm{~s}, 1 \mathrm{H}), 4.01(\mathrm{~s}, 3 \mathrm{H}), 3.86(\mathrm{t}, J=8.4 \mathrm{~Hz}, 2 \mathrm{H}), 3.70(\mathrm{~s}, 3 \mathrm{H}), 3.17(\mathrm{t}, J=8.4 \mathrm{~Hz}$, $2 \mathrm{H}) .{ }^{13} \mathrm{C}$ NMR $\left(100 \mathrm{MHz}, \mathrm{CDCl}_{3}\right): \delta \mathrm{ppm} 167.8,165.6,147.3,142.6,132.4,127.9,125.7,123.1,111.4$, 91.6, 53.3, 51.3, 51.3, 27.4. White solid, m/z: 261.1001 $\left[\mathrm{M}^{+}\right]$. ESI-HRMS Calcd. for $\mathrm{C}_{14} \mathrm{H}_{15} \mathrm{NO}_{4}$ : 262.1079 $[\mathrm{M}+\mathrm{H}]^{+}$, found 262.1079.

17b: ${ }^{1} \mathrm{H}$ NMR (400 MHz, DMSO-d 6 ): $\delta$ ppm $7.75(\mathrm{~m}, 1 \mathrm{H}), 7.61(\mathrm{~m}, 1 \mathrm{H}), 7.34$ (t, J= 7.6 Hz, 1H), 6.66 $(\mathrm{s}, 1 \mathrm{H}), 4.80(\mathrm{t}, J=7.8 \mathrm{~Hz}, 2 \mathrm{H}), 3.93(\mathrm{~s}, 3 \mathrm{H}), 3.48(\mathrm{t}, J=7.6 \mathrm{~Hz}, 2 \mathrm{H}) .{ }^{13} \mathrm{C}$ NMR $\left(100 \mathrm{MHz}, \mathrm{DMSO}-\mathrm{d}_{6}\right)$ : $\delta$ ppm 176.6, 162.4, 144.8, 137.6, 134.5, 127.0, 125.2, 124.1, 120.8, 112.9, 53.2, 52.5, 27.6. Lightyellow solid, m/z: $229.0739\left[\mathrm{M}^{+}\right]$. ESI-HRMS Calcd. for $\mathrm{C}_{13} \mathrm{H}_{11} \mathrm{NO}_{3}: 230.0817[\mathrm{M}+\mathrm{H}]^{+}$, found 230.0816.

17c: ${ }^{1} \mathrm{H}$ NMR $\left(400 \mathrm{MHz}, \mathrm{CDCl}_{3}\right): \delta$ ppm $7.29(\mathrm{~m}, 1 \mathrm{H}), 7.20(\mathrm{~m}, 1 \mathrm{H}), 6.67(\mathrm{~d}, J=8.8 \mathrm{~Hz}, 1 \mathrm{H}), 5.05(\mathrm{~s}$, 1H), $3.99(\mathrm{~s}, 3 \mathrm{H}), 3.87(\mathrm{t}, J=8.4 \mathrm{~Hz}, 2 \mathrm{H}), 3.70(\mathrm{~s}, 3 \mathrm{H}), 3.16(\mathrm{t}, J=8.4 \mathrm{~Hz}, 2 \mathrm{H}) .{ }^{13} \mathrm{C} \mathrm{NMR}(100 \mathrm{MHz}$, $\left.\mathrm{CDCl}_{3}\right): \delta$ ppm 167.6, 165.3, 146.9, 141.9, 134.7, 130.7, 128.8, 115.4, 112.6, 92.7, 53.4, 51.47, 51.4, 27.2. Light-yellow solid, m/z: $339.0106\left[\mathrm{M}^{+}\right]$. ESI-HRMS Calcd. for $\mathrm{C}_{14} \mathrm{H}_{14} \mathrm{BrNO}_{4}: 340.0184[\mathrm{M}+\mathrm{H}]^{+}$, found 340.0181 .

17d: ${ }^{1} \mathrm{H}$ NMR $\left(500 \mathrm{MHz}, \mathrm{D}_{3} \mathrm{CCO}_{2} \mathrm{D}\right): \delta \mathrm{ppm} 8.12(\mathrm{~s}, 1 \mathrm{H}), 7.69(\mathrm{~s}, 1 \mathrm{H}), 7.26(\mathrm{~s}, 1 \mathrm{H}), 4.98(\mathrm{t}, J=7.6 \mathrm{~Hz}$, 2H), $4.02(\mathrm{~s}, 3 \mathrm{H}), 3.57(\mathrm{t}, J=7.6 \mathrm{~Hz}, 2 \mathrm{H})$. Note: DMSO at 2.84. ${ }^{13} \mathrm{C}$ NMR $\left(125 \mathrm{MHz}, \mathrm{D}_{3} \mathrm{CCO}_{2} \mathrm{D}\right): \delta$ ppm 178.7, 163.2, 145.2, 139.6, 138.6, 131.9, 125.7, 125.1, 121.1, 114.8, 55.1, 54.1, 28.5. Off-white solid, m/z: $306.9844\left[\mathrm{M}^{+}\right]$. ESI-HRMS Calcd. for $\mathrm{C}_{13} \mathrm{H}_{10} \mathrm{BrNO}_{3}: 329.9742[\mathrm{M}+\mathrm{Na}]^{+}$, found 329.9736. 18a: No satisfactory NMR data obtained. Yellow solid, m/z: $292.0518\left[\mathrm{M}^{+}\right]$. ESI-HRMS Calcd for $\mathrm{C}_{13} \mathrm{H}_{12} \mathrm{~N}_{2} \mathrm{O}_{4} \mathrm{~S}: 315.0415[\mathrm{M}+\mathrm{Na}]^{+}$, found 315.0418 .

18b: ${ }^{1} \mathrm{H}$ NMR (500 MHz, DMSO-d $)$ ): $\delta$ ppm 12.64 (brs, 1H), 9.44 (s, 1H), 8.41 (d, J=8.8 Hz, $\left.1 \mathrm{H}\right), 8.14$ $(\mathrm{d}, J=8.8 \mathrm{~Hz}, 1 \mathrm{H}), 6.88(\mathrm{~s}, 1 \mathrm{H}), 3.99$ (s, 3H). Note: MeOH at 3.16. ${ }^{13} \mathrm{C}$ NMR $\left(500 \mathrm{MHz}, \mathrm{DMSO}-\mathrm{d}_{6}\right): \delta$ ppm 175.5, 162.5, 157.7, 150.18, 138.3, 137.3, 128.3, 127.7, 120.5, 111.5, 110.3, 53.6. Note: $\mathrm{MeOH}$ at 
48.59. Off-white solid, m/z: $260.0256\left[\mathrm{M}^{+}\right]$. ESI-HRMS Calcd. for $\mathrm{C}_{12} \mathrm{H}_{8} \mathrm{~N}_{2} \mathrm{O}_{3} \mathrm{~S}: 261.0334[\mathrm{M}+\mathrm{H}]^{+}$, found 261.0330 .

19a: ${ }^{1} \mathrm{H}$ NMR $\left(500 \mathrm{MHz}, \mathrm{D}_{3} \mathrm{CCO}_{2} \mathrm{D}\right): \delta$ ppm $9.26(\mathrm{~s}, 1 \mathrm{H}), 8.63(\mathrm{~s}, 1 \mathrm{H}), 8.14(\mathrm{~d}, J=8.7 \mathrm{~Hz}, 1 \mathrm{H}), 7.92$ (d, $J=2.4 \mathrm{~Hz}, 1 \mathrm{H}) 7.48(\mathrm{dd}, J=8.7,2.4 \mathrm{~Hz}, 1 \mathrm{H}), 3.81(\mathrm{~s}, 3 \mathrm{H}), 3.79(\mathrm{~s}, 3 \mathrm{H}) .{ }^{13} \mathrm{C} \mathrm{NMR}(100 \mathrm{MHz}$, $\left.\mathrm{D}_{3} \mathrm{CCO}_{2} \mathrm{D}\right): \delta$ ppm 170.2, 168.2, 157.6, 153.8, 150.3, 138.6, 136.2, 124.7, 118.6, 111.27, 94.2, 52.43, 52.1. Off-white solid, m/z: $292.0518\left[\mathrm{M}^{+}\right]$. ESI-HRMS Calcd. for $\mathrm{C}_{13} \mathrm{H}_{12} \mathrm{~N}_{2} \mathrm{O}_{4} \mathrm{~S}: 315.0415[\mathrm{M}+\mathrm{Na}]^{+}$, found 315.0419 .

19b: ${ }^{1} \mathrm{H}$ NMR $\left(500 \mathrm{MHz}, \mathrm{CF}_{3} \mathrm{CO}_{2} \mathrm{D}\right): \delta \mathrm{ppm} 10.44$ (s, 1H), $9.50(\mathrm{~s}, 1 \mathrm{H}), 8.93(\mathrm{~d}, J=9.3 \mathrm{~Hz}, 1 \mathrm{H}), 8.55$ $(\mathrm{d}, J=9.3 \mathrm{~Hz}, 1 \mathrm{H}), 4.16(\mathrm{~s}, 3 \mathrm{H}) .{ }^{13} \mathrm{C} \mathrm{NMR}\left(500 \mathrm{MHz}, \mathrm{CF}_{3} \mathrm{CO}_{2} \mathrm{D}\right): \delta \mathrm{ppm} 173.6,169.9,166.6,148.9$, 144.7, 142.8, 130.1, 130.0, 125.6, 118.6, 110.2, 56.7. Brown solid, m/z, 260.0256 [M $\left.{ }^{+}\right]$. ESI-HRMS Calcd. for $\mathrm{C}_{12} \mathrm{H}_{8} \mathrm{~N}_{2} \mathrm{O}_{3} \mathrm{~S}: 261.0334[\mathrm{M}+\mathrm{H}]^{+}$, found 261.0331 .

20a: ${ }^{1} \mathrm{H}$ NMR $\left(400 \mathrm{MHz}, \mathrm{CDCl}_{3}\right): \delta$ ppm $11.24(\mathrm{~s}, 1 \mathrm{H}), 7.33(\mathrm{~d}, J=5.4 \mathrm{~Hz}, 1 \mathrm{H}), 6.49(\mathrm{~d}, J=5.4 \mathrm{~Hz}$, $1 \mathrm{H}), 5.49(\mathrm{~s}, 1 \mathrm{H}), 3.91(\mathrm{~s}, 3 \mathrm{H}), 3.82(\mathrm{~s}, 3 \mathrm{H}), 3.78(\mathrm{~s}, 3 \mathrm{H}) .{ }^{13} \mathrm{C} \mathrm{NMR}\left(100 \mathrm{MHz}, \mathrm{CDCl}_{3}\right): \delta \mathrm{ppm} 168.3$, 165.0, 163.9, 145.7, 144.7, 130.8, 119.6, 109.8, 97.4, 53.2, 52.2, 51.8. Yellow solid, m/z: 299.0464 $\left[\mathrm{M}^{+}\right]$. ESI-HRMS Calcd. for $\mathrm{C}_{12} \mathrm{H}_{13} \mathrm{NO}_{6} \mathrm{~S}: 322.0361[\mathrm{M}+\mathrm{Na}]^{+}$, found 322.0360 .

20b: ${ }^{1} \mathrm{H}$ NMR (400 MHz, DMSO-d 6 ): $\delta$ ppm 10.28 (br s, $\left.1 \mathrm{H}\right), 8.79(\mathrm{~s}, 1 \mathrm{H}), 6.46(\mathrm{~d}, J=1.6 \mathrm{~Hz}, 1 \mathrm{H})$, $3.99(\mathrm{~s}, 3 \mathrm{H}) 3.94(\mathrm{~s}, 3 \mathrm{H}) .{ }^{13} \mathrm{C}$ NMR (100 MHz, DMSO-d 6 ): $\delta$ ppm 175.0, 162.7, 162.3, 143.1, 138.4,

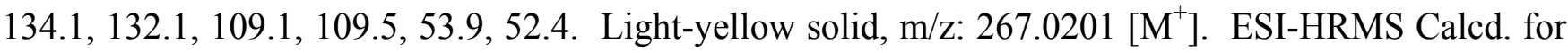
$\mathrm{C}_{11} \mathrm{H}_{9} \mathrm{NO}_{5} \mathrm{~S}: 268.0280[\mathrm{M}+\mathrm{H}]^{+}$, found 268.0282 .

21a: ${ }^{1} \mathrm{H}$ NMR (400 MHz, $\left.\mathrm{CDCl}_{3}\right): \delta$ ppm 12.39 (d, $\left.J=13.7 \mathrm{~Hz}, 1 \mathrm{H}\right), 8.43$ (d, J=13.7 Hz, 1H), 7.51 (d, $J=5.6 \mathrm{~Hz}, 1 \mathrm{H}), 7.17(\mathrm{~d}, J=5.6 \mathrm{~Hz}, 1 \mathrm{H}), 3.93(\mathrm{~s}, 3 \mathrm{H}), 3.90(\mathrm{~s}, 3 \mathrm{H}), 3.78(\mathrm{~s}, 3 \mathrm{H}) .{ }^{13} \mathrm{C} \mathrm{NMR}(100 \mathrm{MHz}$, $\left.\mathrm{CDCl}_{3}\right): \delta$ ppm $167.7,166.1,163.6,150.8,145.4,132.7,116.7,111.5,95.4,52.5,52.1,51.8$. White solid, m/z: $299.0464\left[\mathrm{M}^{+}\right]$. ESI-HRMS Calcd. for $\mathrm{C}_{12} \mathrm{H}_{13} \mathrm{NO}_{6} \mathrm{~S}: 322.0361[\mathrm{M}+\mathrm{Na}]^{+}$, found 322.0355 .

21b: ${ }^{1} \mathrm{H}$ NMR (400 MHz, $\left.\mathrm{D}_{3} \mathrm{CCO}_{2} \mathrm{D}\right): \delta \mathrm{ppm} 8.81(\mathrm{~s}, 1 \mathrm{H}), 8.73(\mathrm{~s}, 1 \mathrm{H}), 3.98(\mathrm{~s}, 3 \mathrm{H}), 3.86(\mathrm{~s}, 3 \mathrm{H}) .{ }^{13} \mathrm{C}$ NMR (100 MHz, $\left.\mathrm{D}_{3} \mathrm{CCO}_{2} \mathrm{D}\right): \delta$ ppm 174.3, 167.2, 163.9, 149.5, 143.46, 135.6, 134.7, 113.4, 110.2, 
53.1, 52.5. Yellow solid, m/z: 267.0201 $\left[\mathrm{M}^{+}\right]$. ESI-HRMS Calcd. for $\mathrm{C}_{11} \mathrm{H}_{9} \mathrm{NO}_{5} \mathrm{~S}: 290.0099[\mathrm{M}+\mathrm{Na}]^{+}$, found 290.0096 .

22a: ${ }^{1} \mathrm{H}$ NMR (400 MHz, $\left.\mathrm{CDCl}_{3}\right): \delta$ ppm $10.03(\mathrm{~s}, 1 \mathrm{H}), 8.26(\mathrm{~d}, J=8.3 \mathrm{~Hz}, 1 \mathrm{H}), 8.16(\mathrm{~d}, J=8.3 \mathrm{~Hz}$, 1H), $7.67-7.61(\mathrm{om}, 3 \mathrm{H}), 6.80(\mathrm{~d}, J=8.0 \mathrm{~Hz}, 1 \mathrm{H}), 5.59(\mathrm{~s}, 1 \mathrm{H}), 3.80(\mathrm{~s}, 3 \mathrm{H}), 3.62(\mathrm{~s}, 3 \mathrm{H}) .{ }^{13} \mathrm{C} \mathrm{NMR}$ $\left(100 \mathrm{MHz}, \mathrm{CDCl}_{3}\right): \delta$ ppm 170.5, 164.7, 149.1, 136.9, 132.7, 129.5, 129.3, 128.1, 127.5, 127.5, 122.8, 119.4, 118.7, 95.2, 53.0, 51.6. Yellow solid, m/z: $363.0106\left[\mathrm{M}^{+}\right]$. ESI-HRMS Calcd. for $\mathrm{C}_{16} \mathrm{H}_{14} \mathrm{BrNO}_{4}$ : $364.0184[\mathrm{M}+\mathrm{H}]^{+}$, found 364.0181

22b: ${ }^{1} \mathrm{H}$ NMR (500 MHz, DMSO-d 6 ): $\delta$ ppm $9.14(\mathrm{dd}, J=8.0,1.6 \mathrm{~Hz}, 1 \mathrm{H}), 8.38(\mathrm{~s}, 1 \mathrm{H}), 8.06(\mathrm{~m}, 1 \mathrm{H})$, $7.70(\mathrm{ddd}, J=8.3,6.8,1.6 \mathrm{~Hz}, 1 \mathrm{H}), 7.64(\mathrm{ddd}, J=8.0,6.8,1.2 \mathrm{~Hz}, 1 \mathrm{H}), 6.80(\mathrm{~s}, 1 \mathrm{H}), 3.85(\mathrm{~s}, 3 \mathrm{H})$. Note: $\mathrm{MeOH}$ at 3.18 (s). ${ }^{13} \mathrm{C}$ NMR $\left(125 \mathrm{MHz}, \mathrm{DMSO}-\mathrm{d}_{6}\right): \delta \mathrm{ppm} 173.5,168.1,147.7,146.5,133.8$, $131.5,127.9,126.58,126.0,126.0,125.4,125.1,115.0,111.5,51.7$. Note: acetone at 30.67. Grey solid, m/z: $330.9844\left[\mathrm{M}^{+}\right]$. ESI-HRMS Calcd. for $\mathrm{C}_{15} \mathrm{H}_{10} \mathrm{BrNO}_{3}: 331.9922[\mathrm{M}+\mathrm{H}]^{+}$, found 331.9926.

23a: ${ }^{1} \mathrm{H} \mathrm{NMR}\left(400 \mathrm{MHz}, \mathrm{CDCl}_{3}\right): \delta$ ppm $10.02(\mathrm{~s}, 1 \mathrm{H}), 8.95(\mathrm{dd}, J=4.0,1.6 \mathrm{~Hz}, 1 \mathrm{H}), 8.48(\mathrm{dd}, J=8.4$, $0.8 \mathrm{~Hz}, 1 \mathrm{H}), 7.92$ (d, J=8.4 Hz,1H), 7.58 (t, $J=7.8 \mathrm{~Hz}, 1 \mathrm{H}), 7.46$ (dd, J=8.4, 4.0 Hz, 1H), 6.99 (dd, J= 7.8, $0.8 \mathrm{~Hz}, 1 \mathrm{H}), 5.59(\mathrm{~s}, 1 \mathrm{H}), 3.78(\mathrm{~s}, 3 \mathrm{H}), 3.58(\mathrm{~s}, 3 \mathrm{H}) . \quad$ Note: acetone at 2.16. ${ }^{13} \mathrm{C} \mathrm{NMR}(100 \mathrm{MHz}$, $\left.\mathrm{CDCl}_{3}\right): \delta \mathrm{ppm} 170.4,164.6,151.0,149.3,149.0,136.9,130.9,129.0,126.9,123.6,121.5,118.9,95.2$ 52.9, 51.6. Note: acetone at 31.07. Yellow solid, m/z: 286.0954 $\left[\mathrm{M}^{+}\right]$. ESI-HRMS Calcd. for $\mathrm{C}_{15} \mathrm{H}_{14} \mathrm{~N}_{2} \mathrm{O}_{4}: 287.1032[\mathrm{M}+\mathrm{H}]^{+}$, found 287.1038.

23b: ${ }^{1} \mathrm{H}$ NMR $\left(500 \mathrm{MHz}, \mathrm{D}_{3} \mathrm{CCO}_{2} \mathrm{D}\right): \delta$ ppm $9.46(\mathrm{~d}, J=8.0 \mathrm{~Hz}, 1 \mathrm{H}), 9.21(\mathrm{dd}, J=4.4 \mathrm{~Hz}, 1.2 \mathrm{~Hz}, 1 \mathrm{H})$, $8.54(\mathrm{~d}, J=9.1 \mathrm{~Hz}, 1 \mathrm{H}), 8.10(\mathrm{~d}, J=8.7 \mathrm{~Hz}, 1 \mathrm{H}), 7.89(\mathrm{dd}, J=8.7 \mathrm{~Hz}, 4.4 \mathrm{~Hz}, 1 \mathrm{H}) 7.56(\mathrm{~s}, 1 \mathrm{H}), 4.09$ (s, 3H). ${ }^{13} \mathrm{C}$ NMR (125 MHz, $\left.\mathrm{D}_{3} \mathrm{CCO}_{2} \mathrm{D}\right): \delta \mathrm{ppm} \mathrm{175.2,} \mathrm{164.8,} \mathrm{151.7,} \mathrm{148.7,} \mathrm{142.0,} \mathrm{140.7,} \mathrm{135.4,} \mathrm{127.5,}$ 125.5, 123.4, 123.0, 122.8, 112.9, 54.4. Off-white solid, m/z: 254.0691 $\left[\mathrm{M}^{+}\right]$. ESI-HRMS Calcd. for $\mathrm{C}_{14} \mathrm{H}_{10} \mathrm{~N}_{2} \mathrm{O}_{3}: 277.0589[\mathrm{M}+\mathrm{Na}]^{+}$, found 277.0586.

24a: ${ }^{1} \mathrm{H}$ NMR (400 MHz, $\left.\mathrm{CDCl}_{3}\right): \delta$ ppm $11.79(\mathrm{~d}, J=12.8 \mathrm{~Hz}, 1 \mathrm{H}), 8.94(\mathrm{~d}, J=4.4,1.6 \mathrm{~Hz}, 1 \mathrm{H}), 8.62$ $(\mathrm{dd}, J=12.8 \mathrm{~Hz}, 1 \mathrm{H}), 8.33(\mathrm{dd}, J=8.4 \mathrm{~Hz}, 1 \mathrm{H}), 7.93(\mathrm{~d}, J=8.4,1 \mathrm{H}), 7.68(\mathrm{t}, J=8.0 \mathrm{~Hz}, 1 \mathrm{H}), 7.47(\mathrm{dd}, J=$

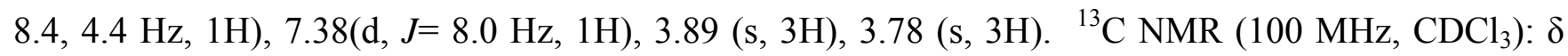


ppm 169.9, 165.7, 153.6, 151.2, 148.8, 135.5, 129.4, 129.3, 127.0, 121.7, 120.7, 113.6, 94.6, 51.9, 51.7. Yellow solid, m/z: $286.0954\left[\mathrm{M}^{+}\right]$. ESI-HRMS Calcd. for $\mathrm{C}_{15} \mathrm{H}_{14} \mathrm{~N}_{2} \mathrm{O}_{4}: 309.0851[\mathrm{M}+\mathrm{Na}]^{+}$, found 309.0852 .

24b: ${ }^{1} \mathrm{H}$ NMR (400 MHz, $\mathrm{CF}_{3} \mathrm{CO}_{2} \mathrm{D}$ ): $\delta$ ppm 10.26 (d, $\left.J=7.6 \mathrm{~Hz}, 1 \mathrm{H}\right), 9.66$ (s, $\left.1 \mathrm{H}\right), 9.52(\mathrm{~s}, 1 \mathrm{H}), 9.12$ $(\mathrm{d}, J=8.4 \mathrm{~Hz}, 1 \mathrm{H}), 8.68(\mathrm{~d}, J=8.4 \mathrm{~Hz}, 1 \mathrm{H}), 8.54(\mathrm{~m}, 1 \mathrm{H}), 4.22(\mathrm{~s}, 3 \mathrm{H}) .{ }^{13} \mathrm{C}$ NMR $(100 \mathrm{MHz}$, $\left.\mathrm{CF}_{3} \mathrm{CO}_{2} \mathrm{D}\right): \delta \mathrm{ppm} 175.4,169.4,149.9,149.4,145.9,143.8,140.1,132.2,127.49,124.4,123.4,122.7$, 111.5, 56.5. Light- brown solid, m/z: $254.0691\left[\mathrm{M}^{+}\right]$. ESI-HRMS Calcd. for $\mathrm{C}_{14} \mathrm{H}_{10} \mathrm{~N}_{2} \mathrm{O}_{3}: 255.0770$ $[\mathrm{M}+\mathrm{H}]^{+}$, found 255.0766.

25a: ${ }^{1} \mathrm{H}$ NMR (400 MHz, $\left.\mathrm{CDCl}_{3}\right): \delta \mathrm{ppm} 10.72(\mathrm{~d}, J=14.0,1 \mathrm{H}), 8.24(\mathrm{~d}, J=14.0 \mathrm{~Hz}, 1 \mathrm{H}), 6.78(\mathrm{~d}$, $J=2.2 \mathrm{~Hz}, 1 \mathrm{H}), 6.70(\mathrm{~d}, J=2.2 \mathrm{~Hz}, 1 \mathrm{H}), 3.89$ (s, 3H), 3.82 (s, 6H), 3.74 (s, 3H). ${ }^{13} \mathrm{C}$ NMR $(100 \mathrm{MHz}$, $\left.\mathrm{CDCl}_{3}\right): \delta \mathrm{ppm} 169.6,166.1,161.2,154.6,125.4,121.9,118.69,107.2,91, .2,51.5,51.4,51.47,37.0$. Off-white solid, m/z: $296.1008\left[\mathrm{M}^{+}\right]$. ESI-HRMS Calcd. for $\mathrm{C}_{13} \mathrm{H}_{16} \mathrm{~N}_{2} \mathrm{O}_{6}: 319.0906[\mathrm{M}+\mathrm{Na}]^{+}$, found 319.0911.

25b: ${ }^{1} \mathrm{H}$ NMR (500 MHz, $\mathrm{CF}_{3} \mathrm{CO}_{2} \mathrm{D}$ ): $\delta$ ppm 9.04 (s, 1H), 7.54 (s, 1H), 4.60 (s, 3H), 4.19 (s, 3H), 4.15 (s, 3H). ${ }^{13} \mathrm{C}$ NMR (125 MHz, $\left.\mathrm{CF}_{3} \mathrm{CO}_{2} \mathrm{D}\right): \delta \mathrm{ppm} 171.2,165.7,164.2,142.9,139.3,138.5,125.2,107.6$, 106.6, 55.9, 55.7, 37.5. Brown solid, m/z: $264.0746\left[\mathrm{M}^{+}\right]$. ESI-HRMS Calcd. for $\mathrm{C}_{12} \mathrm{H}_{12} \mathrm{~N}_{2} \mathrm{O}_{5}$ : $265.0824[\mathrm{M}+\mathrm{H}]^{+}$, found 265.0824.

26a: ${ }^{1} \mathrm{H}$ NMR (400 MHz, $\left.\mathrm{CDCl}_{3}\right): \delta \mathrm{ppm} 9.71(\mathrm{~s}, 1 \mathrm{H}), 7.69(\mathrm{~m}, 2 \mathrm{H}), 6.88,(\mathrm{~m}, 2 \mathrm{H}), 6.81(\mathrm{t}, J=6.0 \mathrm{~Hz}$, $1 \mathrm{H}), 5.53(\mathrm{~s}, 1 \mathrm{H}), 3.76(\mathrm{~s}, 3 \mathrm{H}), 3.74(\mathrm{~s}, 3 \mathrm{H}), 3.72(\mathrm{q}, J=6.0 \mathrm{~Hz}, 2 \mathrm{H}), 2.72(\mathrm{t}, J=6.0,2 \mathrm{H}) .{ }^{13} \mathrm{C}$ NMR $(100$ $\left.\mathrm{MHz}, \mathrm{CDCl}_{3}\right): \delta \mathrm{ppm} 176.4,169.5,166.9,164.4,146.4,143.3,128.9,128.1,119.5,96.3,52.9,51.4$, 35.2, 33.6. Yellow solid, m/z: 350.1114 [M+]. ESI-HRMS Calcd. for $\mathrm{C}_{16} \mathrm{H}_{18} \mathrm{~N}_{2} \mathrm{O}_{7}: 351.1192[\mathrm{M}+\mathrm{H}]^{+}$, found 351.1199 .

26b: ${ }^{1} \mathrm{H}$ NMR (400 MHz, $\left.\mathrm{D}_{3} \mathrm{CCO}_{2} \mathrm{D}\right): \delta$ ppm $8.83(\mathrm{~d}, J=2.0 \mathrm{~Hz}, 1 \mathrm{H}), 8.31$ (dd, $\left.J=8.8 \mathrm{~Hz}, 2.0,1 \mathrm{H}\right), 7.94$ (d, $J=8.8 \mathrm{~Hz}, 1 \mathrm{H}), 7.21(\mathrm{~s}, 1 \mathrm{H}), 4.06(\mathrm{~s}, 3 \mathrm{H}), 3.77$ (t, $J=6.8 \mathrm{HZ}, 2 \mathrm{H}), 3.71(\mathrm{~s}, 3 \mathrm{H}), 2.78(\mathrm{t}, J=6.8 \mathrm{~Hz}$, $2 \mathrm{H}) .{ }^{13} \mathrm{C}$ NMR $\left(100 \mathrm{MHz}, \mathrm{D}_{3} \mathrm{CCO}_{2} \mathrm{D}\right): \delta \mathrm{ppm} 181.5,174.4,168.5,163.6,142.7,139.8,133.9,131.1$, 
125.6, 125.6, 120.9, 112.0, 54.6, 52.6, 37.1, 34.3. Note: DMSO at 39.54. Grey solid, m/z: 332.1008

$\left[\mathrm{M}^{+}\right]$. ESI-HRMS Calcd. for $\mathrm{C}_{16} \mathrm{H}_{16} \mathrm{~N}_{2} \mathrm{O}_{6}: 333.1087[\mathrm{M}+\mathrm{H}]^{+}$, found 333.1097. 